\title{
Clues to a Pd/C catalyst's efficiency can be found on its surface: Identification of an efficient catalyst for the global hydrogenolysis of ether protecting groups
}

\author{
Conor J. Crawford, ${ }^{1,4}$ Yan Qiao, ,2,3 Yequn Liu,, 2,3 Dongmei Huang, ,2,3 Wenjun Yan,, ${ }^{2,3}$ \\ and Stefan Oscarson ${ }^{1, *}$ Shuai Chen,,$^{3, *}$ \\ ${ }^{1}$ Centre for Synthesis and Chemical Biology, University College Dublin, Belfield, Dublin, Ireland. \\ ${ }^{2}$ Center of Materials Science and Optoelectronics Engineering, University of Chinese Academy of \\ Sciences, Beijing 100049, People's Republic of China. \\ ${ }^{3}$ State Key Laboratory of Coal Conversion, Institute of Coal Chemistry, Chinese Academy of Sciences, \\ Taiyuan 030001, People's Republic of China. \\ ${ }^{4}$ C.J.C and Y.Q contributed equally to this work
}

*Corresponding authors, email: chenshuai@sxicc.ac.cn, stefan.oscarson@ucd.ie, qiaoy@sxicc.ac.cn

\begin{abstract}
We report insights into our observations of the wide variability in quality of palladium catalyst from different suppliers, finding the fundamental differences can be rationalized through a combination of $\mathrm{XRD}, \mathrm{XPS}$, and TEM analysis, offering the possibility to predict a catalysts performance prior to the use of valuable synthetic material and save time consuming efforts to identify high quality palladium on carbon catalysts. The synthetic glycan accessed in this study will allow further steps towards the development of semisynthetic vaccines against cryptococcal infections.
\end{abstract}

\section{Introduction}

A major bottleneck we have encountered in accessing pure synthetic glycans is in the final palladium catalyzed hydrogenolysis or global deprotection reactions, where we experienced long reaction times, poor yields, and saturation of aromatic protecting groups to their corresponding ethers. ${ }^{1}$ This was especially prominent in our synthesis of glycans related to structures from $C$. neoformans, ${ }^{2}$ which we carry out in order to develop vaccine candidates. The glucuronoxylomannan (GXM) from native sources is highly heterogenous and large components of the biosynthesis are unknown, meaning synthetic glycans are currently the only viable means to access structures in any meaningful purity. ${ }^{3}$ To overcome this we recently reported a catalyst pre-tuning methodology that increases catalyst selectivity towards hydrogenolysis and inhibits these unwanted saturation by-products. ${ }^{1}$ We also experienced a wide variability in catalyst quality from different manufactures, forcing extensive testing with complex material in order to identify an efficient catalyst, as defined under the parameters of short reaction times, high isolated yields, and its selectivity towards hydrogenolysis over hydrogenation. In order to further advance our understanding and possibly predict a palladium on carbons efficiency prior to use of valuable synthetic material we sought to characterize to the physical properties of the catalysts.

Therefore, we demonstrate clues to a palladium catalysts efficiency can be found by studying the surface chemistry of the catalysts using a combination of high-resolution TEM (HRTEM), X-ray photoelectron spectroscopy (XPS), and X-ray diffractometer (XRD) analysis. Which could prove useful as standards to quickly assess the quality of a catalyst at hand and circumvent the need for extensive optimization experiments with valuable materials from total synthesis. Finally, we prove the analysis of the palladium catalysts completed is useful and mirrors the observed activity during reactions by comparing the activity of these catalysts for deprotecting a serotype A decasaccharide which is currently a candidate for semi-synthetic vaccine development. ${ }^{4}$

\section{Results and Discussion}

Total Synthesis of Serotype A Decasaccharide

To complete the total synthesis of the serotype A decasaccharide we followed a convergent building block approach, utilizing di- and tetrasaccharide thioglycoside building blocks. ${ }^{5}$ The synthesis of which has reported previously, ${ }^{4}$ the use of a convergent synthesis is attractive for several reasons; as it allows quick assembly of the target GXM glycans in minimal steps; the 6-O-acetylation along the mannose backbone is preinstalled; and glycan branching is formed at an early stage, specifically the $\beta-1,2$ xylose branches and $\beta-1,2$ glucuronic acid branches. 


\section{Palladium Catalyst Analysis}

To allow comparison we performed a hydrogenolysis reaction optimisation study using a synthetic serotype A decasaccharide 1, which when deprotected, we have recently identified as a good antigen to be tested as part of a semi-synthetic vaccine candidate against $C$. neoformans infections (Scheme 1 , Table 1$).{ }^{4}$ Using either a Pearman's catalyst $\left(20 \% \mathrm{Pd}[\mathrm{OH}]_{2} / \mathrm{C}\right.$, Sigma-Aldrich) or a $10 \% \mathrm{Pd} / \mathrm{C}$ (SigmaAldrich) both led to exceedingly long reaction times (5-6 days), this was in spite of using our optimised pre-tuning conditions (Table 1 Entry $1 \& 2$ ). While, in keeping with our previous observations the $5 \%$ $\mathrm{Pd} / \mathrm{C}$ Evonik Noblyst ${ }^{\circledR}$ from Strem Chemicals allowed access to the desired decasaccharide 2 in the shortest reaction times, highest yields, and no aromatic protecting group related saturation when using our pre-tuning methodology (Figure 2, Table 1 Entry 3). ${ }^{1}$

While pleased with our findings, we sought to further push our understanding in relation to the wide variability experienced when using different palladium on carbon catalysts. To do so we sought to characterize the catalysts with a range of spectroscopic and imaging techniques. We chose to characterise the three different $\mathrm{Pd} / \mathrm{C}$ catalysts used in this study to allow direct comparison between the isolated yields and reaction times with the direct analysis of the catalysts surface properties. We envisaged that then in the future we could possibly predict a catalysts activity through analysis without the need for extensive optimisation reactions using precious synthetic material.

X-ray diffraction (XRD) analysis of the $5 \% \mathrm{Pd} / \mathrm{C}$ from Strem Chemicals (Figure $1 \mathrm{~A}$ ) obtained diffraction peaks of at $2 \theta$ of $33.3^{\circ}, 34.4^{\circ}, 42.9$ and $55.3^{\circ}$ are assigned to the (002), (101), (110) and (112) facets of tetragonal PdO (powder diffraction file, PDF No. 88-2434), ${ }^{6,7}$ the existence of palladium was confirmed by the peaks at $2 \theta$ of $40.1^{\circ}, 46.7^{\circ}$ and $68.1^{\circ}$, which are correspondent to the (111), (200) and (220) planes of cubic Pd (PDF No. 05-0681) respectively. ${ }^{8-10}$ The broadening and low intensities of the diffraction peaks characteristic of the $5 \% \mathrm{Pd} / \mathrm{C}$ (Strem Chemicals) indicate that the particles are very small. The XRD pattern for the two Sigma-Aldrich catalysts shows clear peaks at $33.3^{\circ}, 34.4^{\circ}, 42.9^{\circ}$ and $55.3^{\circ}$ for crystalline tetragonal PdO, matching well with PDF No. 88-2434. The corresponding XRD peaks intensity from the Sigma-Aldrich catalysts show a significant increase, compared to those of the $5 \% \mathrm{Pd} / \mathrm{C}$ (Strem Chemicals), confirming the size of particle of $\mathrm{PdO}$ is larger in the Sigma-Aldrich catalysts, which is consistent with the TEM image results (Figure 1C). All of the samples show a very broad peak located at $2 \theta$ of $\sim 25^{\circ}$, which assigns to the (002) diffraction planes of graphite microcrystals in the disordered carbon. ${ }^{10,11}$

TEM images were taken of each catalyst and enabled visualisation of the morphology and size distribution of the catalysts (Figure 1C). The $5 \% \mathrm{Pd} / \mathrm{C}$ (Strem Chemicals) indicated that Pd and PdO nanoparticles are uniformly dispersed on carbon black with the mean size of $\sim 4 \mathrm{~nm}$. The existence of large numbers of active sites in the corners and edges of small-sized nanoparticles, is consistent with the observation of a more favourable catalytic performance during the global hydrogenolysis reactions (Table 1 Entry 3 \& 4). The high-resolution TEM (HRTEM) image of the 5\% Pd/C (Strem Chemicals) (Figure 1C) reveals three lattice fringes with space of $0.224 \mathrm{~nm}$ and $0.207 \mathrm{~nm}$, which are corresponding to the (111) crystalline plane of $\mathrm{Pd}$ and (110) crystalline plane of $\mathrm{PdO}$, respectively. This indicates the coexistence of $\mathrm{Pd}$, and $\mathrm{PdO}$ in the $5 \% \mathrm{Pd} / \mathrm{C}$ (Strem Chemicals). Conversely, the catalysts from SigmaAldrich exhibit large particle size and poor size distribution, which is not favourable for catalytic process and corresponds to the lower isolated yields and longer reactions times experienced when using these catalysts (Table 1 Entry $1 \& 2$ ). The HRTEM image of the catalysts from Sigma-Aldrich indicate that the lattice spacing of $\sim 0.208 \mathrm{~nm}$ corresponding to the (110) crystal plane of PdO.

The elemental constituents and states of the catalysts were analysed using XPS analysis, showing high-resolution XPS spectra of Pd $3 d$ (Figure 1B). The binding energies of 337.5 and 342.8 eV were observed in the all catalysts were ascribed to $\mathrm{Pd}^{2+} 3 \mathrm{~d}_{5 / 2}$ and $3 \mathrm{~d}_{3 / 2}$ split orbitals of $\mathrm{PdO}$, respectively. Additionally, in the $5 \% \mathrm{Pd} / \mathrm{C}$ (Strem Chemicals) another two lower binding energies of 336.0 and $341.1 \mathrm{eV}$ are assigned to $3 \mathrm{~d}_{5 / 2}$ and $3 \mathrm{~d}_{3 / 2}$ levels of metallic $\mathrm{Pd}\left(\mathrm{Pd}^{0}\right)$, respectively, ${ }^{12-14}$ again confirming that $\mathrm{PdO}$ and $\mathrm{Pd}$ exist in the $5 \% \mathrm{Pd} / \mathrm{C}$ (Strem Chemicals) but not in the other two catalysts.

\section{Conclusions}

We completed the analysis of three palladium on carbon catalysts physical and chemical properties, finding this can be used to predict a catalysts efficiency prior to the use of precious synthetic materials, as our analysis is consistent with the results of the global deprotections of serotype A decasaccharide in terms of overall reaction efficiency (isolated yields and reaction times). These findings are particularly relevant for complex total synthesis where often access to only minute quantities of material is possible. 


\section{Experimental Section}

\section{General Notes}

Silica gel flash chromatography was carried out using automated flash chromatography systems, Buchi Reveleris ${ }^{\circledR}$ X2 (UV 200-500 nm and ELSD detection, Reveleris ${ }^{\circledR}$ silica cartiges $40 \mu \mathrm{m}$, BÜCHI Labortechnik AG). Size-exclusion chromatography was performed on Bio-Gel® P-2 (Bio-Rad Laboratories Inc.) using isocratic elution $\left(\mathrm{H}_{2} \mathrm{O}: \mathrm{t}^{\mathrm{BuOH}}, 99: 1, \mathrm{v} / \mathrm{v}\right)$. Instrumentation: peristaltic pump P-3 (Pharmacia Fine Chemicals), refractive index detector lota 2 (Precision Instruments), PrepFC fraction collector (Gilson Inc.). Software: Trilution $\AA$ LC (version 1.4, Gilson Inc.). All chemicals for the synthesis were purchased from commercial suppliers (Acros, Carbosynth Ltd, Fisher Scientific Ltd, A/S, Merck, Sigma-Aldrich, VWR, Strem Chemicals and AlfaAesar) and used without purification. Dry Solvents were obtained from a PureSolv-EN $N^{T M}$ solvent purification system (Innovative Technology Inc.). All other anhydrous solvents were used as purchased from Sigma-Aldrich in AcroSeal® bottles.

\section{Procedure for Catalyst Pre-treatment ${ }^{1}$}

$500 \mathrm{mg} \mathrm{Pd} / \mathrm{C}$ (any commercial catalyst), was suspended in $1 \mathrm{~mL} \mathrm{DMF:} \mathrm{H}_{2} \mathrm{O}$ mixture $(80: 20 \mathrm{v} / \mathrm{v})$, and the solution was made acidic by the addition of $200 \mu \mathrm{L} \mathrm{HCl}$ (ACS Reagent, $37 \%, \mathrm{pH} 2-3$ ), with or without an atmosphere of hydrogen gas for about 20 minutes. The presence of dimethylamine was confirmed via ninhydrin staining. The treated $\mathrm{Pd} / \mathrm{C}$ catalysts was re-isolated though filtration. The moistened catalyst was then be used directly in the hydrogenolysis reaction.

\section{General Procedure for Hydrogenolysis Batch Reaction ${ }^{1}$}

The treated catalyst (0.2-0.5 molar eq. of palladium per benzyl group) was added to a solution of oligosaccharide (1 eq.) dissolved in THF:tert-butyl alcohol:PBS (100 mM, pH 4) $(60: 10: 30, \mathrm{v} / \mathrm{v} / \mathrm{v})$. The reaction was placed in a high pressure reactor at 10 bar and was monitored via normal phase TLC ( $\mathrm{MeCN}: \mathrm{H}_{2} \mathrm{O}$ mixtures) and MALDI-TOF mass spectrometry Once complete the reaction mixture was filtered through a plug of Ceilte ${ }^{\circledR}$ and then concentrated in vacuo. The residue was then re-dissolved in a minimal amount of sterile water and purified with a Bio-gel P2 Column, after lyophilization to yield the desired product.

\section{Palladium on Carbon Characterization}

A transmission electron microscope (JEOL JEM-2100F) was used to obtain transmission electron microscopy (TEM) images, high-resolution TEM (HRTEM) images at an acceleration voltage of $200 \mathrm{kV}$. An X-ray diffractometer (XRD, Bruker D8 Advance) with Cu- $\mathrm{K}_{\alpha}$ radiation was used for analyzing the crystallographic structure of the as-prepared samples from $5^{\circ} \sim 90^{\circ}$ with a scanning step of $0.02^{\circ}$. The surface elemental composition and chemical state of the as-prepared samples were collected from an X-ray photoelectron spectra spectrometer (XPS, Kratos AXIS ULTRA DLD), in which a monochromatic Al $\mathrm{K}_{\alpha}$ source $(\mathrm{hv}=1486.6 \mathrm{eV})$ was applied. All binding energies were calibrated using the $\mathrm{C}_{1 \mathrm{~s}}$ hydrocarbon peak at $284.60 \mathrm{eV}$. 


\section{Acknowledgements}

We thank Dr Yannick Ortin and Dr Jimmy Muldoon for NMR and MS support. C.J.C. was funded by Irish Research Council postgraduate award (GOIPG/2016/998). S.O was supported by Science Foundation Ireland Award 13/IA/1959.

\section{References}

1 C. Crawford and S. Oscarson, European J. Org. Chem., 2020, 2020, 3332-3337.

2 C. Crawford, M. P. Wear, D. F. Q. Smith, C. D'Errico, A. Casadevall and S. Oscarson, ChemRxiv, , DOI:10.26434/CHEMRXIV.12144699.V1.

3 C. J. Crawford, R. J. B. Cordero, L. Guazzelli, M. P. Wear, A. Bowen, S. Oscarson and A. Casadevall, J. Biol. Chem., 2020, 295, 4327-4340.

4 L. Guazzelli, C. Crawford, R. Ulc, A. Bowen, O. McCabe, A. J. Jedlicka, M. P. Wear, A. Casadevall and S. Oscarson, Chem. Sci., 2020, DOI:10.1039/D0SC01249A.

5 L. Guazzelli, R. Ulc, L. Rydner and S. Oscarson, Org. Biomol. Chem., 2015, 13, 6598-6610.

6 W. Hu, G. X. Li, J. J. Chen, F. J. Huang, Y. Wu, S. D. Yuan, L. Zhong and Y. Q. Chen, Chem. Commun., 2017, 53, 6160-6163.

7 H. J. Cho, V. T. Chen, S. Qiao, W. T. Koo, R. M. Penner and I. D. Kim, ACS Sensors, $2018,3,2152-2158$.

8 J. Zhao, W. Jing, T. Tan, X. Liu, Y. Kang and W. Wang, New J. Chem., 2020, 44, 4604-4612.

P. Xi, F. Chen, G. Xie, C. Ma, H. Liu, C. Shao, J. Wang, Z. Xu, X. Xu and Z. Zeng, Nanoscale, 2012, 4, 5597-5601.

10 L. Lan, F. Du and C. Xia, RSC Adv., 2016, 6, 109023-109029.

11 W. Xu, C. Lv, Y. Zou, J. Ren, X. She, Y. K. Zhu, Y. Zhang, S. Chen, X. Yang, T. Zhan, J. Sun and D. Yang, J. Power Sources, 2019, 442, 227184.

S. Meher and R. K. Rana, Green Chem., 2019, 21, 2494-2503.

J. Lv, S. Wu, Z. Tian, Y. Ye, J. Liu and C. Liang, J. Mater. Chem. A, 2019, 7, 12627-12634.

L. Han, Q. Li, S. Chen, W. Xie, W. Bao, L. Chang and J. Wang, Sci. Rep., 2017, DOI:10.1038/s41598-017-07802-8. 
Scheme 1. Global Deprotection of Serotype A Decasaccharide

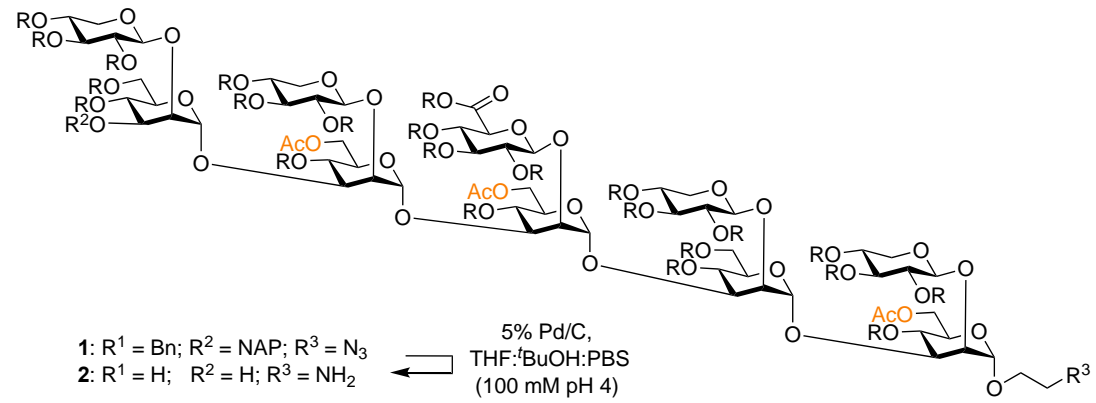

Table 1. Global Deprotection of GXM Glycans ${ }^{a}$

\begin{tabular}{cccccc}
\hline Entry & Substrate & Catalyst & Supplier & Time $(\mathrm{d})$ & $\%$ Yield \\
\hline 1 & $\mathbf{1}$ & $20 \% \mathrm{Pd}[\mathrm{OH}]_{2}$ & Sigma-Aldrich & 6 & 76 \\
2 & $\mathbf{1}$ & $10 \% \mathrm{Pd} / \mathrm{C}$ & Sigma-Aldrich & 5 & 58 \\
3 & $\mathbf{1}$ & $5 \% \mathrm{Pd} / \mathrm{C}$ & Strem Chemicals & 1.5 & 88
\end{tabular}

a Preconditioned catalyst (see protocol), THF: $\mathrm{BuOH}: \mathrm{PBS}(100 \mathrm{mM} \mathrm{pH}$ 4) $(60: 10: 30 \mathrm{v} / \mathrm{v} / \mathrm{v})$. 
A

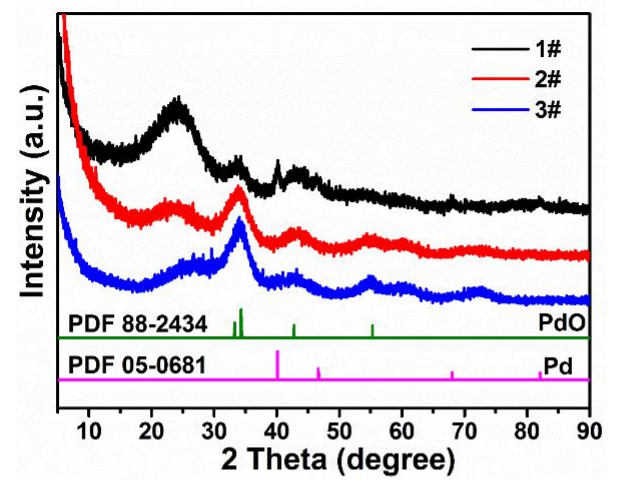

B

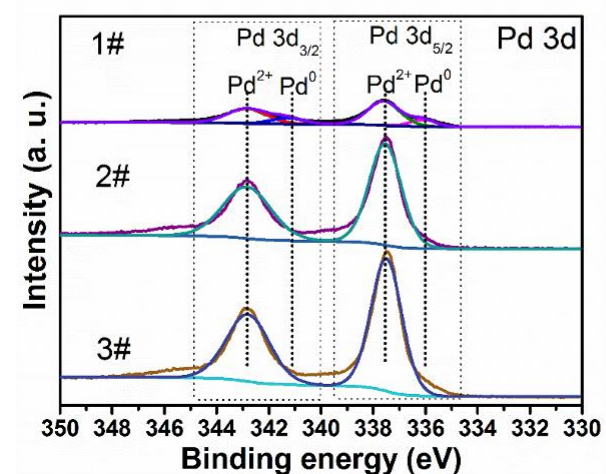

C

TEM
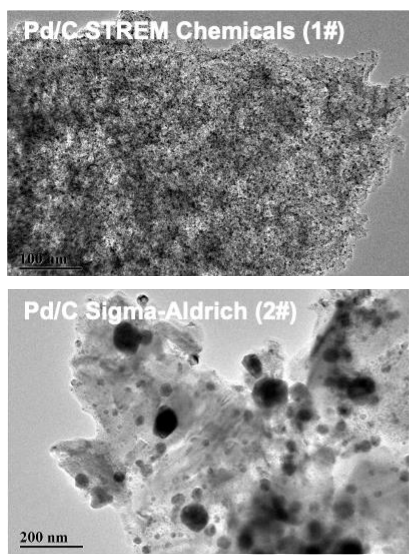

$\mathrm{Pd}\left[\mathrm{O} \mathrm{J}_{2} / \mathrm{C}\right.$ Sigma-Aldrich(s:)

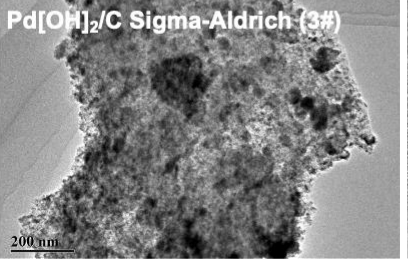

HRTEM
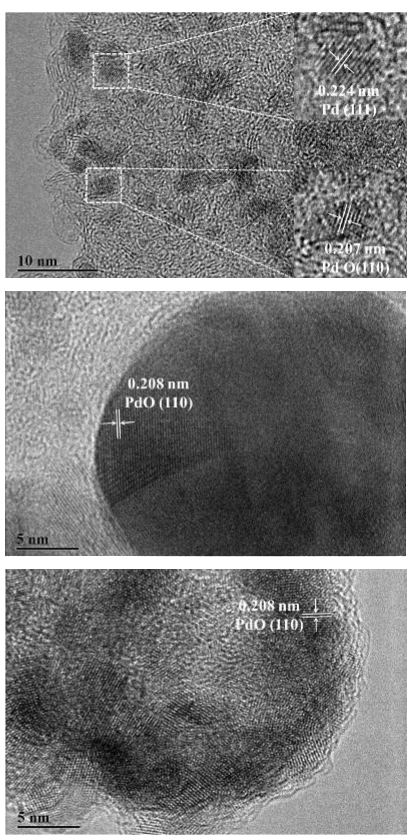

Figure 1. Characterization of Palladium on Carbon Catalysts. Pd/C STREM Chemicals (\#1), $P d / C$ Sigma-Aldrich (\#2), and $\mathrm{Pd}[\mathrm{OH}]_{2} / \mathrm{C}$ Sigma-Aldrich (3\#). A. XRD patterns of the Pd/C. B. XPS for Pd $3 d$ electrons. C. TEM and HRTEM of catalyst. Scale inset. 


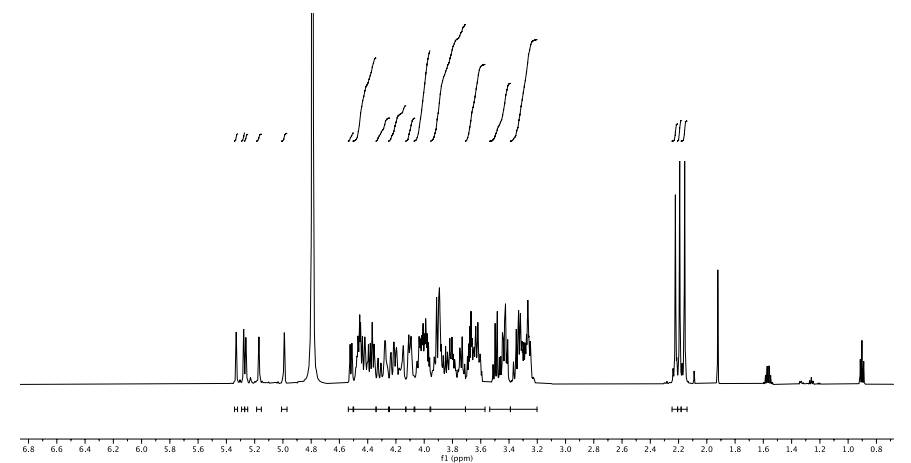

Figure 2. ${ }^{1} \mathrm{H}$ NMR Spectrum of Serotype A Decasaccharide. 